Article

\title{
The Asymmetry is Derived from Mechanical Interlocking of Achiral Axle and Achiral Ring Components -Syntheses and Properties of Optically Pure [2]Rotaxanes-
}

\author{
Keiji Hirose ${ }^{1, *}$ (D), Masaya Ukimi ${ }^{1}$, Shota Ueda ${ }^{1}$, Chie Onoda $^{1}$, Ryohei Kano ${ }^{1}$, \\ Kyosuke Tsuda ${ }^{1}$, Yuko Hinohara ${ }^{1}$ and Yoshito Tobe ${ }^{2}$ \\ 1 Graduate School of Engineering Science, Osaka University, 1-3 Machikaneyama, Toyonaka, \\ Osaka 560-8531, Japan; ukimim@supra.chem.es.osaka-u.ac.jp (M.U.); \\ uedas@supra.chem.es.osaka-u.ac.jp (S.U.); onodat@supra.chem.es.osaka-u.ac.jp (C.O.); \\ kanor@supra.chem.es.osaka-u.ac.jp (R.K.); tsudak@supra.chem.es.osaka-u.ac.jp (K.T.); \\ hinoharay@supra.chem.es.osaka-u.ac.jp (Y.H.) \\ 2 The Institute of Scientific and Industrial Research, Osaka University, 8-1 Mihogaoka, Ibaraki, \\ Osaka 567-0047, Japan; tobe@chem.es.osaka-u.ac.jp \\ * Correspondence: hirose@chem.es.osaka-u.ac.jp; Tel.: +81-6-6850-6228
}

Received: 25 November 2017; Accepted: 27 December 2017; Published: 9 January 2018

\begin{abstract}
Rotaxanes consisting of achiral axle and achiral ring components can possess supramolecular chirality due to their unique geometrical architectures. To synthesize such chiral rotaxanes, we adapted a prerotaxane method based on aminolysis of a metacyclophane type prerotaxane that had planar chirality, which is composed of an achiral stopper unit and a crown ether type ring component. The prerotaxanes were well resolved using chiral HPLC into a pair of enantiomerically pure prerotaxanes, which were transferred into corresponding chiral rotaxanes, respectively. Obtained chiral rotaxanes were revealed to have considerable enantioselectivity.
\end{abstract}

Keywords: mechanical chirality; mecanostereochemistery; supramolecular chirality; rotaxane; aminolysis

\section{Introduction}

The discoveries of a crown ether and a very stable complex formation with a metal cation by C. J. Pedersen [1] in 1967, following neologisms of the "Host-guest chemistry" by D. J. Cram [2], and that of the "Supramolecular chemistry" by J. M. Lehn [3], showed the importance of interaction between molecules, were tremendously influential and initiated a huge amount of researches. Nobel prize of chemistry was awarded to these researchers in 1987. Many crops from this field of chemistry were industrialized such as ion sensors, ion selective membranes, and electrodes [4-9], which were contributed by ion recognition initiated by C. J. Pedersen and chiral selectors for chromatography [10-15] initiated by the contribution of D. J. Cram's chirality recognition technology $[16,17]$. Research topics such as chiral shift reagents for the determination of enantiomeric excess of chiral substance [18], besides the reagents for the determination of absolute configuration $[19,20]$, chiral indicators $[19,21-24]$, and rapid chirality detection method using chiral hosts by means of mass spectrometry $[25,26]$, as well as other well established methods [27-29] have long fascinated many researchers.

In the late 1980s, fabrications of molecular assemblies using inter- and/or intramolecular interactions of molecular components came under the spotlight. Typical examples are the syntheses of catenanes, rotaxanes, and molecular knots [30]. The emergence and excellent development of supramolecular methods make the syntheses of supramolecules much more effective, with a higher 
chemical yield [31-35] than before [36]. Covalent methods [37], which were not thought to be versatile for the synthesis of interlocked molecules, were also well developed [38,39]. One of the applications in this field of chemistry is to make the smallest machines at molecular level using fabrications of molecular components. The Nobel prize in chemistry was awarded to three researchers working in this field: J.-P. Sauvage [40], Sir J. F. Stoddart [41], and B. L. Feringa [42] in 2016.

Chiral interlocked molecules provide unique three-dimensional, valuable-binding circumstances capable of chirality recognition of guests. However, the application of interlocked hosts for chirality recognition has been largely overlooked, especially the recognition by hosts with mechanical chirality. A rotaxane has a mechanically interlocked architecture consisting of a dumbbell-shaped axle component that is threaded through a ring component. Rotaxanes consisting of achiral axle components that have $C_{\infty \mathrm{v}}$ symmetry and achiral ring components that have $C_{\mathrm{s}}$ symmetry can be chiral, caused by their geometrically specific architectures. In Figure 1, an enantiomeric pair of rotaxanes with the specific chirality composed of a $C_{\infty \mathrm{v}}$ axle and a $C_{\mathrm{s}}$ ring components is shown. Rotaxanes with the specific chirality can be expected as new molecular platforms [43-45] for asymmetric catalysts, chiral sensors [27-29], etc. However, it is difficult to synthesize such rotaxanes as optically pure forms [46]. In order to synthesize such rotaxanes with the rotaxane-specific chirality in an optically pure form, we applied our prerotaxane method [39], in which a rotaxane can be synthesized via backside attack of a stopper unit to a prerotaxane with planar chirality composed of an achiral stopper unit and a $C_{\mathrm{s}}$ ring component (Scheme 1). In order to investigate binding properties of the rotaxanes that have a mechanically interlocked chirality as host compounds, precise evaluation of the enantioselective complexation ability of the rotaxanes with chiral guests were carried out.

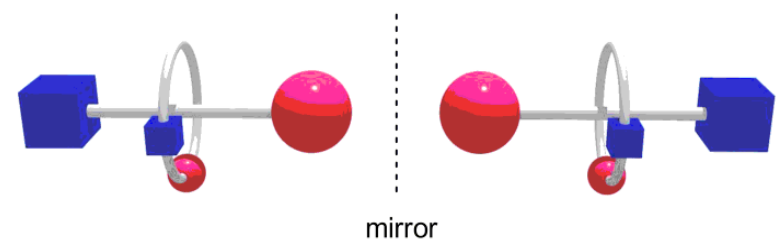

Figure 1. Rotaxane specific chirality by a combination of $C_{\infty \mathrm{v}}$ axle component and $C_{\mathrm{s}}$ ring component.

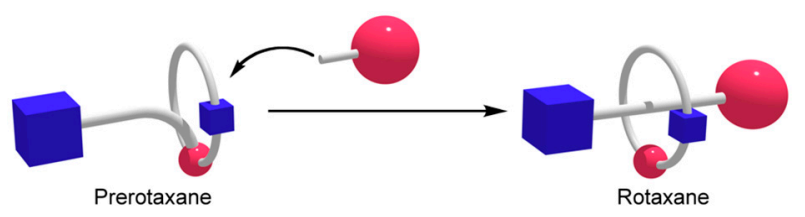

Scheme 1. Key step of our rotaxane synthesis of covalent method via $\mathrm{S}_{\mathrm{N}} 2$ reaction with a prerotaxane with planar chirality and stopper unit.

\section{Materials and Methods}

Compound 1 prepared using hydroquinone mono-methoxymethyl ether as starting material according to previously reported procedures [47-49] was used. All other compounds and reagents were obtained from commercial suppliers and used as received. $\mathrm{CH}_{2} \mathrm{Cl}_{2}$ and THF were dried by a Glass Contour solvent purification system prior to use. Melting points were measured with a hot-stage apparatus equipped with a thermometer. ${ }^{1} \mathrm{H}$ NMR spectra were recorded with a JEOL GSX-270, a Varian Mercury 300, or a JEOL AL-400 spectrometer for solutions in $\mathrm{CDCl}_{3}$ or $\mathrm{C}_{6} \mathrm{D}_{6}$ with $\mathrm{SiMe}_{4}$ as an internal standard and $J$ values given in $\mathrm{Hz} .{ }^{13} \mathrm{C}$ NMR spectra were recorded at $75.5 \mathrm{MHz}$ with a JEOL GSX-270 spectrometer, and chloroform ( $\delta \mathrm{C} 77.0)$ was used as a chemical shift reference. Multiplicities for ${ }^{1} \mathrm{H}$ NMR spectra are as follows: singlet (s), doublet (d), triplet $(\mathrm{t})$, quartet (q), and multiplet (m). Multiplicities for ${ }^{13} \mathrm{C}$ NMR spectra are as follows: primary $\left(1^{\circ}\right)$, secondary $\left(2^{\circ}\right)$, tertiary $\left(3^{\circ}\right)$, and quaternary $\left(4^{\circ}\right)$. IR spectra were measured on a JASCO FT/IR-410 spectrophotometer. Circular dichroism spectra were measured using a JASCO J-805 spectropolarimeter, 
and $\theta$ values are given in units of mdeg. MS spectra were recorded with a JEOL JMS-700 spectrometer. Preparative GPLC was performed with JAI LC-908 on JAIGEL $1 \mathrm{H}$ and $2 \mathrm{H}$ columns with $\mathrm{CHCl}_{3}$ as a solvent. Elemental analyses were carried out by using a Perkin-Elmer 2400II analyser. Analytical thin layer chromatography (TLC) was performed using precoated silica gel plates (Merck Kieselgel 60 F254). Preparative column chromatography was carried out using Fuji Silysia BW-300 silica gel $\left(\mathrm{SiO}_{2}\right.$; $0.038-0.075 \mathrm{~mm}$ ) with the indicated solvents, which were mixed $v / v$ as specified.

\section{2-1 Synthetic Procedures and Characterization of Racemic Prerotaxanes (rac)-3}

Into a solution of $1(98.9 \mathrm{mg}, 142 \mu \mathrm{mol})$ in dry THF $(3.5 \mathrm{~mL})$ and $\mathrm{KO}^{t} \mathrm{Bu}(55.6 \mathrm{mg}, 495 \mu \mathrm{mol})$, 3,5-dinitrobenzoyl chloride $(131 \mathrm{mg}, 568 \mu \mathrm{mol})$ was added with stirring under a nitrogen atmosphere. After $2 \mathrm{~h}$ stirring at room temperature, the solvent was evaporated under reduced pressure. The residue was extracted with chloroform. The organic layer was washed with brine. After being dried over anhydrous $\mathrm{MgSO}_{4}$, the solvent was removed under reduced pressure. The residue was purified by preparative GPLC to give (rac)-3 $(74.8 \mathrm{mg}, 84.4 \mu \mathrm{mol}, 60 \%)$ as an orange powder: ${ }^{1} \mathrm{H}$ NMR $(400 \mathrm{MHz}$, $\left.\mathrm{CDCl}_{3}, 30^{\circ} \mathrm{C}\right) \delta 9.02(\mathrm{~d}, 2 \mathrm{H}, J=1.9 \mathrm{~Hz}), 8.86(\mathrm{~d}, 1 \mathrm{H}, J=2.5 \mathrm{~Hz}), 8.81(\mathrm{t}, 1 \mathrm{H}, J=1.9 \mathrm{~Hz}), 8.58(\mathrm{dd}, 1 \mathrm{H}$, $J=2.5,8.8 \mathrm{~Hz}), 8.28(\mathrm{~d}, 1 \mathrm{H}, J=2.5 \mathrm{~Hz}), 8.16(\mathrm{~d}, 1 \mathrm{H}, J=2.5 \mathrm{~Hz}), 7.85(\mathrm{~d}, 1 \mathrm{H}, J=8.8 \mathrm{~Hz}), 7.45(\mathrm{~d}, 2 \mathrm{H}$, $J=8.5 \mathrm{~Hz}), 7.18-7.30(\mathrm{~m}, 2 \mathrm{H}), 7.09(\mathrm{~s}, 1 \mathrm{H}), 6.85(\mathrm{~s}, 1 \mathrm{H}), 5.45(\mathrm{~d}, 1 \mathrm{H}, J=12 \mathrm{~Hz}), 5.15(\mathrm{~d}, 1 \mathrm{H}, J=12 \mathrm{~Hz})$, $4.83(\mathrm{~d}, 1 \mathrm{H}, J=13 \mathrm{~Hz}), 4.70(\mathrm{~d}, 1 \mathrm{H}, J=13 \mathrm{~Hz}), 4.08-4.18(\mathrm{~m}, 2 \mathrm{H}), 3.92(\mathrm{t}, 2 \mathrm{H}, J=4.2 \mathrm{~Hz}), 3.48-3.78$ $(\mathrm{m}, 16 \mathrm{H}) ;{ }^{13} \mathrm{C} \mathrm{NMR}\left(100 \mathrm{MHz}, \mathrm{CDCl}_{3}, 30^{\circ} \mathrm{C}\right) \delta 159.9\left(4^{\circ}\right), 151.2\left(4^{\circ}\right), 150.3\left(4^{\circ}\right), 148.9\left(4^{\circ}\right), 148.7\left(4^{\circ}\right)$, $147.84\left(4^{\circ}\right), 147.77\left(4^{\circ}\right), 146.5\left(4^{\circ}\right), 134.2\left(4^{\circ}\right), 131.3\left(4^{\circ}\right), 129.5\left(3^{\circ}\right), 129.2\left(4^{\circ}\right), 128.5\left(4^{\circ}\right), 127.9\left(3^{\circ}\right), 126.4$ $\left(3^{\circ}\right), 126.0\left(3^{\circ}\right), 125.9\left(3^{\circ}\right), 125.1\left(3^{\circ}\right), 124.8\left(3^{\circ}\right), 124.4\left(3^{\circ}\right), 122.0\left(3^{\circ}\right), 120.19\left(3^{\circ}\right), 122.16\left(3^{\circ}\right), 109.2\left(3^{\circ}\right)$, $107.7\left(3^{\circ}\right), 71.3\left(2^{\circ}\right), 71.0\left(2^{\circ}\right), 70.8\left(2^{\circ}\right), 70.71\left(2^{\circ}\right), 70.65\left(2^{\circ}\right), 70.6\left(2^{\circ}\right), 70.1\left(2^{\circ}\right), 69.4\left(2^{\circ}\right), 69.0\left(2^{\circ}\right)$, $68.5\left(2^{\circ}\right), 67.7\left(2^{\circ}\right)$; IR (KBr) 3093, 2922, 2866, 1770, 1543, 1345, 1261, 1137, 1114, 920, $717 \mathrm{~cm}^{-1}$; HRMS (FAB) $m / z$ calcd for $\mathrm{C}_{41} \mathrm{H}_{39} \mathrm{~N}_{6} \mathrm{O}_{17}\left(\left[\mathrm{M}+\mathrm{H}^{+}\right]\right)$: 887.2372, found: 887.2379.

\section{2-2 Characterization of Resolved Prerotaxane 3 st}

3 1st: m.p. $88.8-90.1{ }^{\circ} \mathrm{C} ;{ }^{1} \mathrm{H}$ NMR $\left(400 \mathrm{MHz}, \mathrm{CDCl}_{3}, 30{ }^{\circ} \mathrm{C}\right) \delta 9.02(\mathrm{~d}, 2 \mathrm{H}, J=1.9 \mathrm{~Hz}), 8.86(\mathrm{~d}, 1 \mathrm{H}$, $J=2.5 \mathrm{~Hz}), 8.81(\mathrm{t}, 1 \mathrm{H}, J=1.9 \mathrm{~Hz}), 8.58(\mathrm{dd}, 1 \mathrm{H}, J=2.5,8.8 \mathrm{~Hz}), 8.28(\mathrm{~d}, 1 \mathrm{H}, J=2.5 \mathrm{~Hz}), 8.16(\mathrm{~d}, 1 \mathrm{H}$, $J=2.5 \mathrm{~Hz}), 7.85(\mathrm{~d}, 1 \mathrm{H}, J=8.8 \mathrm{~Hz}), 7.45(\mathrm{~d}, 2 \mathrm{H}, J=8.5 \mathrm{~Hz}), 7.18-7.30(\mathrm{~m}, 2 \mathrm{H}), 7.09(\mathrm{~s}, 1 \mathrm{H}), 6.85$ $(\mathrm{s}, 1 \mathrm{H}), 5.45(\mathrm{~d}, 1 \mathrm{H}, J=12 \mathrm{~Hz}), 5.15(\mathrm{~d}, 1 \mathrm{H}, J=12 \mathrm{~Hz}), 4.83(\mathrm{~d}, 1 \mathrm{H}, J=13 \mathrm{~Hz}), 4.70(\mathrm{~d}, 1 \mathrm{H}, J=13 \mathrm{~Hz})$, $4.08-4.18(\mathrm{~m}, 2 \mathrm{H}), 3.92(\mathrm{t}, 2 \mathrm{H}, J=4.2 \mathrm{~Hz}), 3.48-3.78(\mathrm{~m}, 16 \mathrm{H}) ;{ }^{13} \mathrm{C} \mathrm{NMR}\left(100 \mathrm{MHz}, \mathrm{CDCl}_{3}, 30{ }^{\circ} \mathrm{C}\right)$ ઈ $160.0\left(4^{\circ}\right), 151.3\left(4^{\circ}\right), 150.4\left(4^{\circ}\right), 149.0\left(4^{\circ}\right), 148.8\left(4^{\circ}\right), 147.94\left(4^{\circ}\right), 147.89\left(4^{\circ}\right), 146.6\left(4^{\circ}\right), 134.2\left(4^{\circ}\right)$, $131.4\left(4^{\circ}\right), 129.6\left(3^{\circ}\right), 129.2\left(4^{\circ}\right), 128.6\left(4^{\circ}\right), 128.0\left(3^{\circ}\right), 126.5\left(3^{\circ}\right), 126.1\left(3^{\circ}\right), 126.0\left(3^{\circ}\right), 125.2\left(3^{\circ}\right), 124.9$ $\left(3^{\circ}\right), 124.5\left(3^{\circ}\right), 122.1\left(3^{\circ}\right), 120.29\left(3^{\circ}\right), 120.25\left(3^{\circ}\right), 109.2\left(3^{\circ}\right), 107.7\left(3^{\circ}\right), 71.3\left(2^{\circ}\right), 71.0\left(2^{\circ}\right), 70.77\left(2^{\circ}\right)$, $70.75\left(2^{\circ}\right), 70.73\left(2^{\circ}\right), 70.66\left(2^{\circ}\right), 70.63\left(2^{\circ}\right), 70.1\left(2^{\circ}\right), 69.5\left(2^{\circ}\right), 69.0\left(2^{\circ}\right), 68.5\left(2^{\circ}\right), 67.7\left(2^{\circ}\right)(1$ tertiary carbon and 1 quaternary carbon could not be seen); IR (KBr) 3101, 2869, 1752, 1543, 1344, 1261, 1137, 1114, 922, $717 \mathrm{~cm}^{-1}$; UV/Vis $\left(\mathrm{CHCl}_{3}, 22{ }^{\circ} \mathrm{C}\right) \lambda_{\max }(\log \varepsilon) 342$ (4.3); MS (LDI) $\mathrm{m} / z$ 909.3 ([M + $\left.\left.\mathrm{Na}^{+}\right]\right)$. HRMS (ESI) $m / z$ Calcd for $\mathrm{C}_{41} \mathrm{H}_{38} \mathrm{~N}_{6} \mathrm{NaO}_{17}$ : 909.2191, Found: 909.2200 ([M+ Na $\left.\left.{ }^{+}\right]\right)$. Anal. Calcd for $\mathrm{C}_{41} \mathrm{H}_{38} \mathrm{~N}_{6} \mathrm{O}_{17}$ : C, 55.53; $\mathrm{H}, 4.32 ; \mathrm{N}, 9.48$. Found: $\mathrm{C}, 55.25 ; \mathrm{H}, 4.22 ; \mathrm{N}, 9.35$.

\section{2-3 Characterization of Resolved Prerotaxane 3 2nd}

3 2nd $_{\text {n.p. }}$ 88.9-90.0 ${ }^{\circ} \mathrm{C} ;{ }^{1} \mathrm{H}$ NMR $\left(400 \mathrm{MHz}, \mathrm{CDCl}_{3}, 30^{\circ} \mathrm{C}\right) \delta 9.02(\mathrm{~d}, 2 \mathrm{H}, J=1.9 \mathrm{~Hz}), 8.86(\mathrm{~d}, 1 \mathrm{H}$, $J=2.5 \mathrm{~Hz}), 8.81(\mathrm{t}, 1 \mathrm{H}, J=1.9 \mathrm{~Hz}), 8.58(\mathrm{dd}, 1 \mathrm{H}, J=2.5,8.8 \mathrm{~Hz}), 8.28(\mathrm{~d}, 1 \mathrm{H}, J=2.5 \mathrm{~Hz}), 8.16(\mathrm{~d}, 1 \mathrm{H}$, $J=2.5 \mathrm{~Hz}), 7.85(\mathrm{~d}, 1 \mathrm{H}, J=8.8 \mathrm{~Hz}), 7.45(\mathrm{~d}, 2 \mathrm{H}, J=8.5 \mathrm{~Hz}), 7.18-7.30(\mathrm{~m}, 2 \mathrm{H}), 7.09(\mathrm{~s}, 1 \mathrm{H}), 6.85(\mathrm{~s}, 1 \mathrm{H})$, $5.45(\mathrm{~d}, 1 \mathrm{H}, J=12 \mathrm{~Hz}), 5.15(\mathrm{~d}, 1 \mathrm{H}, J=12 \mathrm{~Hz}), 4.83(\mathrm{~d}, 1 \mathrm{H}, J=13 \mathrm{~Hz}), 4.70(\mathrm{~d}, 1 \mathrm{H}, J=13 \mathrm{~Hz}), 4.08-4.18$ $(\mathrm{m}, 2 \mathrm{H}), 3.92(\mathrm{t}, 2 \mathrm{H}, J=4.2 \mathrm{~Hz}), 3.48-3.78(\mathrm{~m}, 16 \mathrm{H}) ;{ }^{13} \mathrm{C} \mathrm{NMR}\left(100 \mathrm{MHz}, \mathrm{CDCl}_{3}, 30{ }^{\circ} \mathrm{C}\right) \delta 160.0,151.3$, 150.4, 149.0, 148.8, 147.94, 147.89, 146.6, 134.2, 131.4, 129.6, 129.2, 128.6, 128.0, 126.5, 126.1, 126.0, 125.2, $124.9,124.5,122.1,120.3,109.2,107.7,71.3,71.1,70.79,70.77,70.74,70.69,70.64,70.2,69.5,69.0,68.5$, 67.8 ( 2 carbons couldn't be seen); IR (KBr) 3101, 2870, 1752, 1543, 1345, 1261, 1138, 1114, 922, $718 \mathrm{~cm}^{-1}$; MS (LDI) $m / z$ 909.2 ([M + Na $\left.{ }^{+}\right]$). HRMS (ESI) $m / z$ Calcd for $\mathrm{C}_{41} \mathrm{H}_{38} \mathrm{~N}_{6} \mathrm{NaO}_{17}$ : 909.2191 , Found: 909.2185 $\left(\left[\mathrm{M}+\mathrm{Na}^{+}\right]\right)$. Anal. Calcd for $\mathrm{C}_{41} \mathrm{H}_{38} \mathrm{~N}_{6} \mathrm{O}_{17}: \mathrm{C}, 55.53 ; \mathrm{H}, 4.32 ; \mathrm{N}, 9.48$. Found: $\mathrm{C}, 55.70 ; \mathrm{H}, 4.46 ; \mathrm{N}, 9.35$. 


\section{2-4 Synthetic Procedures and Characterization of Rotaxanes $5_{1 \text { st }}$}

Into a solution of $3_{1 s t}(2.99 \mathrm{mg}, 3.37 \mu \mathrm{mol})$ in $\mathrm{C}_{6} \mathrm{D}_{6}(660 \mu \mathrm{L})$, 3,5-bis(trifluoromethyl)benzylamine $4(1.63 \mathrm{mg}, 6.71 \mu \mathrm{mol})$ in $\mathrm{C}_{6} \mathrm{D}_{6}(22 \mu \mathrm{L})$ was added. The aminolysis was monitored by ${ }^{1} \mathrm{H}$ NMR $(270 \mathrm{MHz})$ at $30{ }^{\circ} \mathrm{C}$. After the reaction was completed, the solvent was removed under reduced pressure. The residue was purified by preparative GPLC. Following precipitation from $\mathrm{CH}_{2} \mathrm{Cl}_{2}$ and hexane afforded $5_{1 \mathrm{st}}(2.93 \mathrm{mg}, 2.59 \mu \mathrm{mol}, 77 \%)$ as an orange solid. m.p. $115.1-116.8^{\circ} \mathrm{C} ;{ }^{1} \mathrm{H} \mathrm{NMR}$ $\left(270 \mathrm{MHz}, \mathrm{C}_{6} \mathrm{D}_{6}, 30{ }^{\circ} \mathrm{C}\right) \delta 8.69(\mathrm{~d}, 2 \mathrm{H}, J=1.7 \mathrm{~Hz}), 8.40(\mathrm{t}, 1 \mathrm{H}, J=1.7 \mathrm{~Hz}), 8.27-8.24(\mathrm{~m}, 1 \mathrm{H}), 8.20$ $(\mathrm{s}, 2 \mathrm{H}), 8.07(\mathrm{~d}, 1 \mathrm{H}, J=2.2 \mathrm{~Hz}), 8.05(\mathrm{~d}, 1 \mathrm{H}, J=2.4 \mathrm{~Hz}), 7.66(\mathrm{~s}, 1 \mathrm{H}), 7.62(\mathrm{dd}, 1 \mathrm{H}, J=8.3,2.4 \mathrm{~Hz}), 7.60$ $(\mathrm{d}, 1 \mathrm{H}, J=2.4 \mathrm{~Hz}), 7.37(\mathrm{~d}, 1 \mathrm{H}, J=9.0 \mathrm{~Hz}), 7.45-7.33(\mathrm{~m}, 4 \mathrm{H}), 6.96(\mathrm{~s}, 1 \mathrm{H}), 6.03(\mathrm{~s}, 1 \mathrm{H}), 5.34(\mathrm{dd}, 1 \mathrm{H}$, $J=16.2,8.0 \mathrm{~Hz}), 5.20(\mathrm{~d}, 1 \mathrm{H}, J=8.8 \mathrm{~Hz}), 4.89(\mathrm{dd}, 1 \mathrm{H}, J=16.2,2.3 \mathrm{~Hz}), 4.71(\mathrm{~d}, 1 \mathrm{H}, J=10.6 \mathrm{~Hz})$, $4.37(\mathrm{~d}, 1 \mathrm{H}, J=9.1 \mathrm{~Hz}), 3.78(\mathrm{~d}, 1 \mathrm{H}, J=10.6 \mathrm{~Hz}), 3.61-2.91(\mathrm{~m}, 16 \mathrm{H}), 2.69(\mathrm{t}, 2 \mathrm{H}, J=10.2 \mathrm{~Hz}), 2.43$ $(\mathrm{d}, 1 \mathrm{H}, J=10.7 \mathrm{~Hz}), 2.12(\mathrm{t}, 1 \mathrm{H}, J=10.1 \mathrm{~Hz}) ;{ }^{13} \mathrm{C} \mathrm{NMR}\left(100 \mathrm{MHz}, \mathrm{CDCl}_{3}, 30^{\circ} \mathrm{C}\right) \delta 163.0\left(4^{\circ}\right), 160.2$ $\left(4^{\circ}\right), 148.7\left(4^{\circ}\right), 147.6\left(4^{\circ}\right), 147.4\left(4^{\circ}\right), 147.2\left(4^{\circ}\right), 146.8\left(4^{\circ}\right), 146.0\left(4^{\circ}\right), 140.7\left(4^{\circ}\right), 137.2\left(4^{\circ}\right), 132.0\left(3^{\circ}\right)$, $130.3\left(3^{\circ}\right), 130.2\left(\mathrm{q}, J_{\mathrm{CF}}=33 \mathrm{~Hz}, 4^{\circ}\right), 128.9\left(4^{\circ}\right), 128.4\left(4^{\circ}\right), 127.8\left(3^{\circ}\right), 127.65\left(3^{\circ}\right), 127.59\left(4^{\circ}\right), 126.5\left(3^{\circ}\right)$, $125.6\left(3^{\circ}\right), 124.92\left(4^{\circ}\right), 124.90\left(3^{\circ}\right), 124.86\left(3^{\circ}\right), 124.7\left(3^{\circ}\right), 122.1\left(4^{\circ}\right), 120.4\left(\mathrm{q}, J_{\mathrm{CF}}=4.4 \mathrm{~Hz}, 3^{\circ}\right), 120.0\left(3^{\circ}\right)$, $119.8\left(3^{\circ}\right), 119.7\left(3^{\circ}\right), 107.3\left(3^{\circ}\right), 105.8\left(3^{\circ}\right), 71.20\left(2^{\circ}\right), 71.15\left(2^{\circ}\right), 70.9\left(2^{\circ}\right), 70.6\left(2^{\circ}\right), 70.5\left(2^{\circ}\right), 70.4\left(2^{\circ}\right)$, $70.2\left(2^{\circ}\right), 70.1\left(2^{\circ}\right), 69.8\left(2^{\circ}\right), 69.4\left(2^{\circ}\right), 67.6\left(2^{\circ}\right), 65.1\left(2^{\circ}\right), 43.7\left(2^{\circ}\right)(2$ tertiary carbon and 3 quaternary carbon could not be seen); IR (KBr) 3346, 3096, 2880, 1601, 1540, 1345, 1278, 1130, $850 \mathrm{~cm}^{-1}$; UV/Vis $\left(\mathrm{CHCl}_{3}, 20^{\circ} \mathrm{C}\right) \lambda_{\max }(\log \varepsilon) 390$ (4.3); MS (MALDI) $\mathrm{m} / \mathrm{z} 1152.4$ ([M + $\left.\mathrm{Na}^{+}\right]$); HRMS (FAB) $\mathrm{m} / z \mathrm{Calcd}$ for $\mathrm{C}_{51} \mathrm{H}_{45} \mathrm{~F}_{6} \mathrm{~N}_{7} \mathrm{O}_{17}: 1130.2854$, Found: $1130.2885\left(\left[\mathrm{M}+\mathrm{H}^{+}\right]\right)$.

\section{2-5 Synthetic Procedures and Characterization of Rotaxanes $\boldsymbol{5}_{\mathbf{2 n d}}$}

Into a solution of $\mathbf{3}_{2 \mathrm{nd}}(3.15 \mathrm{mg}, 3.54 \mu \mathrm{mol})$ in $\mathrm{C}_{6} \mathrm{D}_{6}(660 \mu \mathrm{L})$, amine $4(1.72 \mathrm{mg}, 7.07 \mu \mathrm{mol})$ in $\mathrm{C}_{6} \mathrm{D}_{6}$ $(22 \mu \mathrm{L})$ was added. The aminolysis was monitored by ${ }^{1} \mathrm{H}$ NMR $(270 \mathrm{MHz})$ at $30{ }^{\circ} \mathrm{C}$. After the reaction was completed, the solvent was removed under reduced pressure. The residue was purified by preparative GPLC. Following precipitation from $\mathrm{CH}_{2} \mathrm{Cl}_{2}$ and hexane afforded $\boldsymbol{5}_{2 \mathrm{nd}}(3.35 \mathrm{mg}, 2.96 \mu \mathrm{mol}$, $84 \%)$ as an orange solid. m.p. $115.2-116.7^{\circ} \mathrm{C} ;{ }^{1} \mathrm{H} \mathrm{NMR}\left(400 \mathrm{MHz}, \mathrm{C}_{6} \mathrm{D}_{6}, 30^{\circ} \mathrm{C}\right) \delta 8.69(\mathrm{~d}, 2 \mathrm{H}, J=1.7 \mathrm{~Hz})$, $8.40(\mathrm{t}, 1 \mathrm{H}, J=1.7 \mathrm{~Hz}), 8.26-8.25(\mathrm{~m}, 1 \mathrm{H}), 8.20(\mathrm{~s}, 2 \mathrm{H}), 8.07(\mathrm{~d}, 1 \mathrm{H}, J=2.0 \mathrm{~Hz}), 8.05(\mathrm{~d}, 1 \mathrm{H}, J=2.5 \mathrm{~Hz})$, $7.66(\mathrm{~s}, 1 \mathrm{H}), 7.62(\mathrm{dd}, 1 \mathrm{H}, J=8.3,2.4 \mathrm{~Hz}), 7.60(\mathrm{~d}, 1 \mathrm{H}, J=2.4 \mathrm{~Hz}), 7.37(\mathrm{~d}, 1 \mathrm{H}, J=9.0 \mathrm{~Hz}), 7.45-7.33$ $(\mathrm{m}, 4 \mathrm{H}), 6.96(\mathrm{~s}, 1 \mathrm{H}), 6.03(\mathrm{~s}, 1 \mathrm{H}), 5.34(\mathrm{dd}, 1 \mathrm{H}, J=16.2,8.0 \mathrm{~Hz}), 5.20(\mathrm{~d}, 1 \mathrm{H}, J=9.5 \mathrm{~Hz}), 4.89(\mathrm{dd}, 1 \mathrm{H}$, $J=14.3,2.3 \mathrm{~Hz}), 4.71(\mathrm{~d}, 1 \mathrm{H}, J=10.3 \mathrm{~Hz}), 4.37(\mathrm{~d}, 1 \mathrm{H}, J=9.5 \mathrm{~Hz}), 3.79(\mathrm{~d}, 1 \mathrm{H}, J=10.5 \mathrm{~Hz}), 3.60-2.91$ $(\mathrm{m}, 16 \mathrm{H}), 2.68(\mathrm{t}, 2 \mathrm{H}, J=10.2 \mathrm{~Hz}), 2.43(\mathrm{~d}, 1 \mathrm{H}, J=10.7 \mathrm{~Hz}), 2.13(\mathrm{t}, 1 \mathrm{H}, J=10.1 \mathrm{~Hz}) ;{ }^{13} \mathrm{C} \mathrm{NMR}(100 \mathrm{MHz}$, $\left.\mathrm{CDCl}_{3}, 30^{\circ} \mathrm{C}\right) \delta 163.0,160.2,148.7,147.6,147.4,147.2,146.8,146.0,140.7,137.2,132.0,130.3,130.2$ $\left(\mathrm{q}, J_{C F}=33 \mathrm{~Hz}\right), 128.9,128.4,127.8,127.65,127.59,126.5,125.6,124.92,124.90,124.86,124.7,122.1,120.4$ $\left(\mathrm{q}, J_{C F}=4.4 \mathrm{~Hz}\right), 120.0,119.8,119.7,107.3,105.8,71.20,71.15,70.9,70.6,70.5,70.4,70.2,70.1,69.8,69.4$, 67.6, 65.1, 43.7 (2 tertiary carbon and 3 quaternary carbon could not be seen); IR (KBr) 3351, 3101, 2880, 1601, 1540, 1345, 1278, 1131, $850 \mathrm{~cm}^{-1}$; MS (MALDI) m/z 1152.3 ([M + $\left.\mathrm{Na}^{+}\right]$); HRMS (FAB) $\mathrm{m} / \mathrm{z}$ Calcd for $\mathrm{C}_{51} \mathrm{H}_{45} \mathrm{~F}_{6} \mathrm{~N}_{7} \mathrm{O}_{17}$ : 1130.2854, Found: $1130.2852\left(\left[\mathrm{M}+\mathrm{H}^{+}\right]\right)$.

\section{Results and Discussion}

We planned to synthesize rotaxanes 5 and the enantiomer as target chiral rotaxanes by way of prerotaxanes 3 with planar chirality followed by aminolysis with bulky amine 4 as shown in Scheme 2 . This versatile covalent method to synthesize rotaxane consists of two steps: first, esterification of a phenolic crown ether with an acid chloride; second, aminolysis with an amine compound having a bulky group.

Prerotaxanes 3 were prepared from a phenolic pseudo-crown ether 1 and a 3,5-dinitrobenzoyl chloride in the presence of $\mathrm{KO}^{t} \mathrm{Bu}$ in THF. Optical resolution of obtained racemic prerotaxane (rac)-3 was performed by preparative chiral HPLC on Daicel CHIRALFLASH IA. Chromatograms of (rac)-3, resolved $\mathbf{3}_{1 \mathrm{st}}$, and $\boldsymbol{3}_{2 \text { nd }}$ were shown in Figure 2. (rac)-3 was well resolved into $\boldsymbol{3}_{1 \mathrm{st}}$ and $\boldsymbol{3}_{2 \text { nd }}$. In Figure 3 , CD (a) and UV-visible (b) spectra of $\boldsymbol{3}_{1 \text { st }}$ and $\boldsymbol{3}_{2 \text { nd }}$ are shown. The CD spectra of $\boldsymbol{3}_{1 \mathrm{st}}$ and $\boldsymbol{3}_{2 \text { nd }}$ are mirror 
images each other. In general, chirality that appeared in mechanically interlocked systems is attractive due to its unique structure having large and flexible asymmetric field owing to the high mobility of the components. However, the determination of the absolute configuration is therefore difficult when applying chromophore sector rules on CD spectal data [50,51]. Because absolute structures of these enantiomers $\mathbf{3}$ were not determined in this stage, resolved prerotaxanes were denoted as $\boldsymbol{3}_{1 \text { st }}$ and $\boldsymbol{3}_{2 \text { nd }}$ according to the eluted order in this condition.
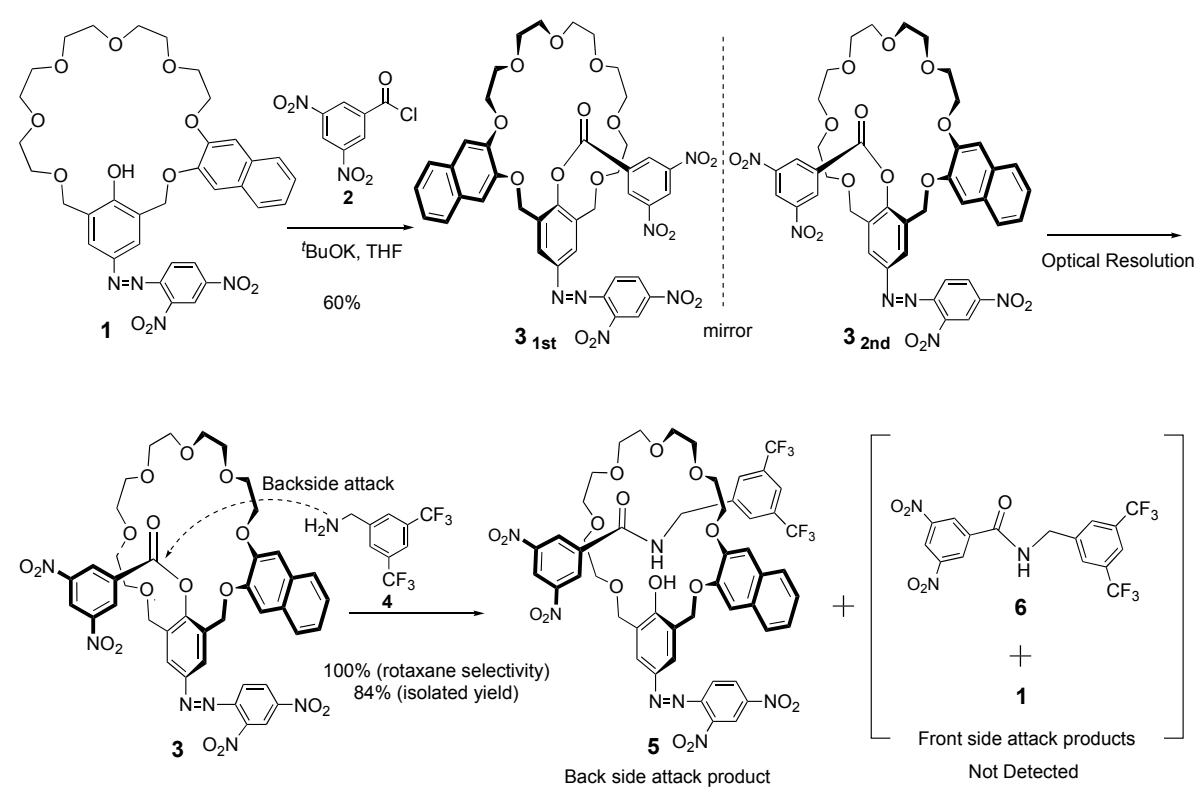

Scheme 2. Synthetic scheme of 5 via aminolysis of prerotaxane 3.

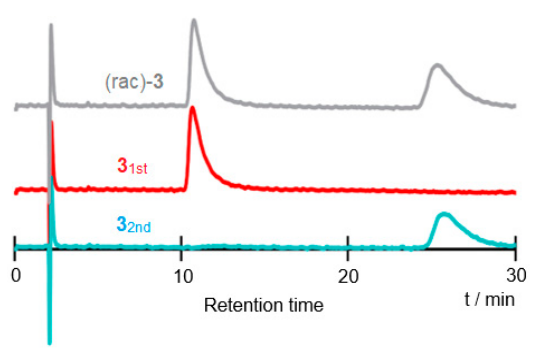

Figure 2. HPLC chromatogram of prerotaxane (rac)-3, and resolved $\mathbf{3}_{1 \text { st }}$ and $\mathbf{3}_{2 \text { nd }}$, detected by UV at $330 \mathrm{~nm}$. (Conditions; Column: DAICEL CHIRALPAK IA (10 $\mathrm{mm} \varphi \times 250 \mathrm{~mm})$; mobile phase: hexane $/$ dichloromethane $=50 / 50$; flow rate $=1.0 \mathrm{~mL} / \mathrm{min}$; temperature: $30^{\circ} \mathrm{C}$ ).

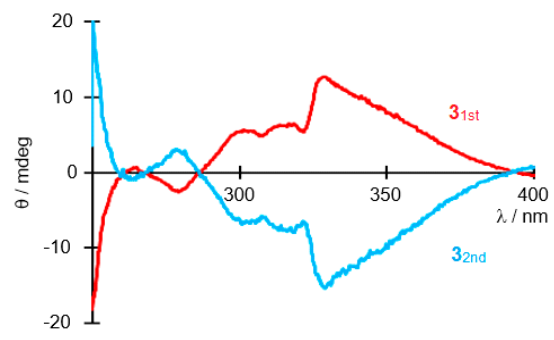

(a)

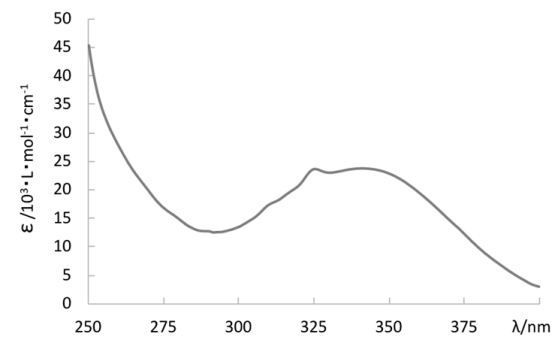

(b)

Figure 3. (a) Circular dichroism spectra of enantiomerically pure prerotaxanes $3_{1 \text { st }}$ (red) and $3_{2 \text { nd }}$ (blue) in $\mathrm{CHCl}_{3}$ at room temperature $\left(\left[3_{1 \mathrm{st}}\right]=65.9 \mu \mathrm{M},\left[3_{2 \mathrm{nd}}\right]=76.1 \mu \mathrm{M}\right.$, cell length $\left.=1.0 \mathrm{~cm}\right) ;(\mathbf{b}) \mathrm{Uv}$-visible spectrum of $\boldsymbol{3}_{2 \text { nd }}$ under same condition. 
For preparation of optically pure rotaxane 5 , aminolyses of corresponding enantiomeric pure prerotaxanes 3 were carried out. A mixture of a prerotaxane 3 and amine 4 in benzene was stirred at room temperature. Because the aminolysis proceeds via the nucleophilic attack of amino group of 4 from the backside of the crown ether ring of prerotaxanes 3 as shown with dotted arrow in Scheme 2, the corresponding rotaxanes $\mathbf{5}$ were afforded from a pair of enantiomerically pure prerotaxanes $\mathbf{3}$, respectively. The reactions of the resolved prerotaxanes $3_{1 \text { st }}$ and $3_{2 \text { nd }}$ with amine 4 proceeded quantitatively in $\mathrm{C}_{6} \mathrm{D}_{6}$ to give corresponding rotaxanes $5_{1 s t}$ and $5_{2 \text { nd }}$, respectively. The residue after removal of the solvent in the reaction mixture was subjected to GPLC and/or column chromatography on silica gel to give rotaxane $5_{1 \text { st }}$ and $5_{2 \text { nd }}$ without producing any dumbbell compound $\mathbf{6}$ and crown ether 1 . The efficiency of the reaction was easily monitored by ${ }^{1} \mathrm{H}$ NMR. The aminolysis by the backside attack of amine 4 took place selectively. ${ }^{1} \mathrm{H}$ NMR spectra of a reaction mixture of aminolysis of 3 are shown in Figure 4. $\mathrm{H}^{\mathrm{A}}$ signal of prerotaxane $3\left(\mathrm{H}^{\mathrm{A}}(3)\right)$ at $9.02 \mathrm{ppm}$ decreased with increasing $\mathrm{H}^{\mathrm{A}}$ signal of rotaxane $5\left(\mathrm{H}^{\mathrm{A}}(5)\right)$ at $8.69 \mathrm{ppm}$ without producing any ring compound 1, e.g., $\mathrm{H}^{\mathrm{a}}$ and $\mathrm{H}^{\mathrm{b}}$ signals at 5.02 and $4.60 \mathrm{ppm}$ were not observed. Reaction proceeded fast $\left(\mathrm{t}_{1 / 2}: 20 \mathrm{~min}\right)$ and selectively (rotaxane selectivity: $>99 \%$ ). Actually, $\mathbf{5}$ was obtained in high isolated yield $(84 \%)$.
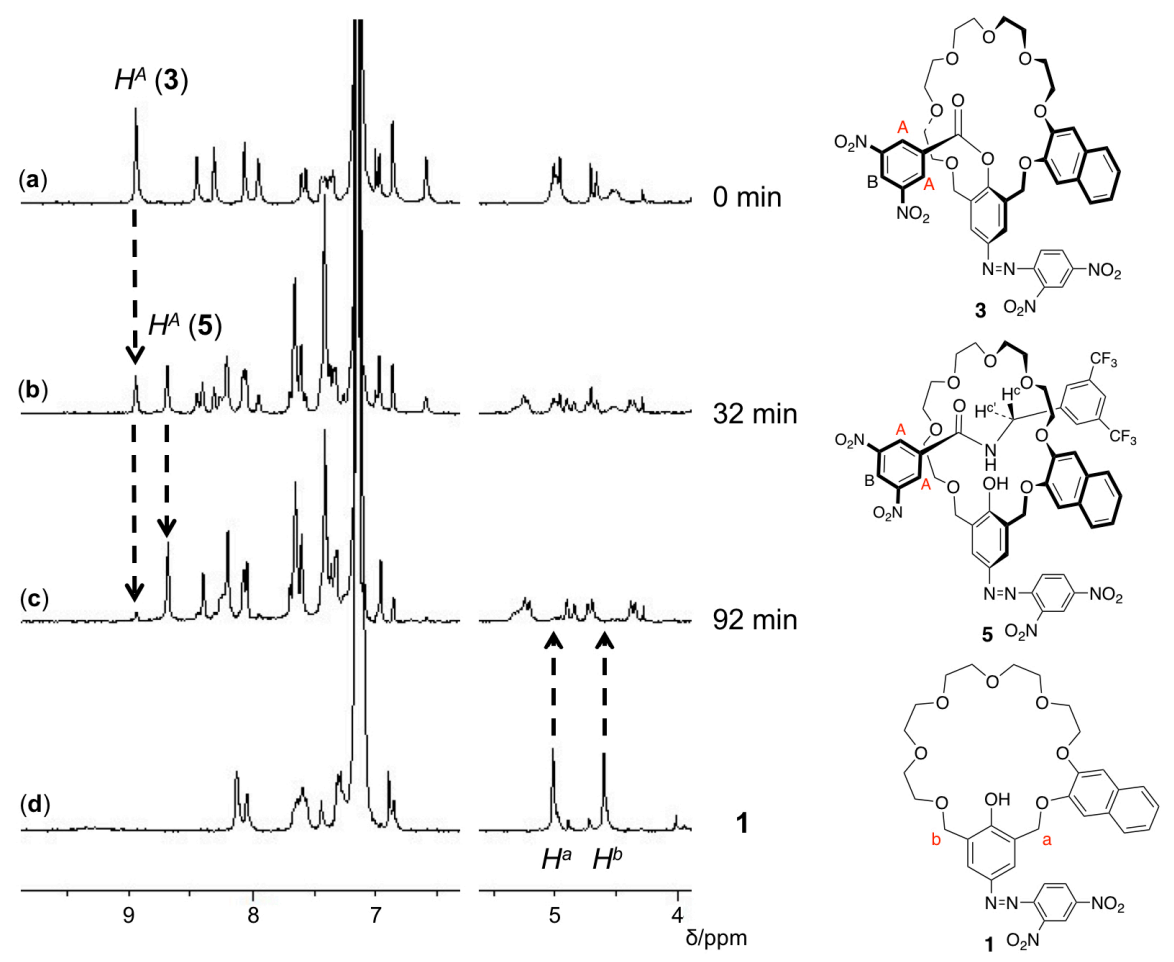

Figure 4. ${ }^{1} \mathrm{H}$ NMR $\left(270 \mathrm{MHz}, \mathrm{C}_{6} \mathrm{D}_{6}\right)$ spectra of (a) reaction mixture at $0 \mathrm{~min}$ (prerotaxane $3_{2 \mathrm{nd}}$ ), (b) $32 \mathrm{~min}$, (c) $92 \mathrm{~min}$, and (d) ring 1 for reference. The descriptors refer to the signals representing rotaxane $\left(\mathrm{H}^{\mathrm{A}}(5)\right)$, dumbbell $\left(\mathrm{H}^{\mathrm{A}}(\mathbf{6})\right)$, and ring1 $\left(\mathrm{H}^{\mathrm{a}}\right.$ and $\left.\mathrm{H}^{\mathrm{b}}\right)$ protons are shown in Scheme 1 .

The structures of rotaxanes 5 were characterized by spectral data. ${ }^{1} \mathrm{H}$ NMR spectra of rotaxane 5 and the corresponding dumbbell $\mathbf{6}$ are shown in Figure 5. Significant downfield shift of the signal of the amide proton $\mathrm{NH}$ of the axle in rotaxanes 5 was observed relative to the corresponding signal of dumbbell $\mathbf{6}$ ( $\delta 6.83$ to 8.25 ), indicating the formation of hydrogen bonding between the amide hydrogen with the oxygen atoms of the crown ether moiety of 5 . Two singlets assigned to the benzylic protons $\mathrm{H}^{\mathrm{a}}$ and $\mathrm{H}^{\mathrm{b}}$ of the crown ether became double double-doublets $\left(\mathrm{H}^{\mathrm{a}}, \mathrm{H}^{\mathrm{a}^{\prime}}, \mathrm{H}^{\mathrm{b}}\right.$, and $\left.\mathrm{H}^{\mathrm{b}^{\prime}}\right)$, evidencing that the ring components are threaded and interlocked by the dumbbell component 6 with different stoppers. More importantly, signals of homotopic methylene protons $\mathrm{H}^{\mathrm{C}}$ on the axle component became multiplet, because the methylene protons $\mathrm{H}^{\mathrm{C}}$ became diastereotopic in the chiral circumstance 
of the rotaxane structure-specific chirality generated by interlocking with $C_{\mathrm{s}}$ ring component as shown in Figure 5.

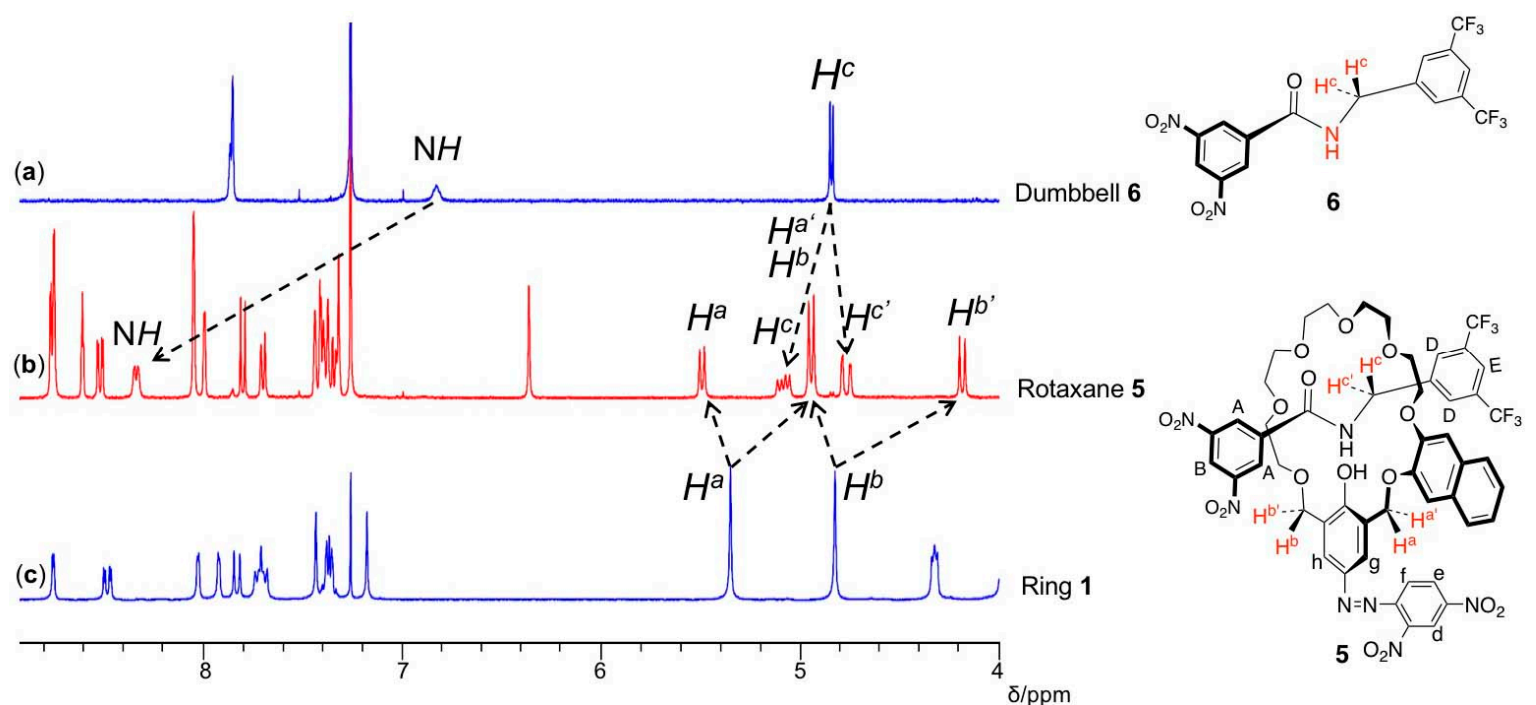

Figure 5. ${ }^{1} \mathrm{H}$ NMR spectra of dumbbell $\mathbf{6}(\mathbf{a})$, chiral rotaxane $\mathbf{5}(\mathbf{b})$, and ring $\mathbf{1}(\mathbf{c})$ in $\mathrm{CDCl}_{3}$ at $30{ }^{\circ} \mathrm{C}$ (400 MHz).

HPLC Chromatograms on Daicel CHIRALPAK IA shown in Figure 6 with the mirror images of the CD spectra shown in Figure $7 \mathrm{a}$ evidenced that obtained $\boldsymbol{5}_{1 \mathrm{st}}$ and $\boldsymbol{5}_{\mathbf{2 n d}}$ are pure enantiomers.

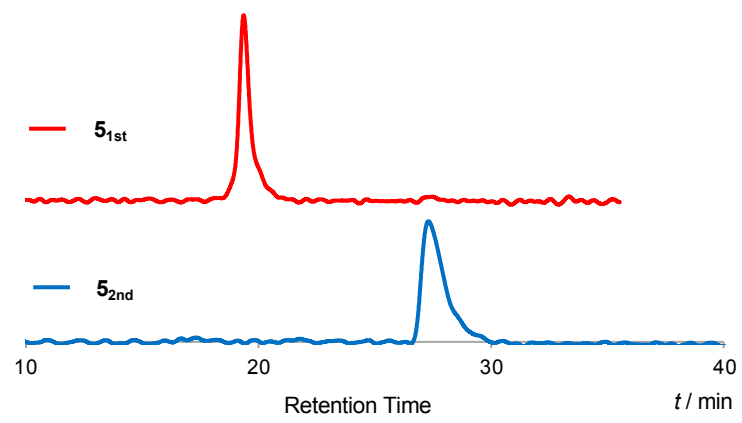

Figure 6 . HPLC chromatograms of rotaxanes $\boldsymbol{5}_{1 \mathrm{st}}$ (red), $\boldsymbol{5}_{2 \text { nd }}$ (blue) detected by UV at $254 \mathrm{~nm}$. (Column: CHIRALPAK IA (4.6 $\mathrm{mm} \varphi \times 150 \mathrm{~mm}$ ); mobile phase: hexane/dichloromethane /trifluoroacetic acid $=80 / 20 / 0.1$; flow rate $=0.8 \mathrm{~mL} / \mathrm{min}$; temperature: $30^{\circ} \mathrm{C}$ ).

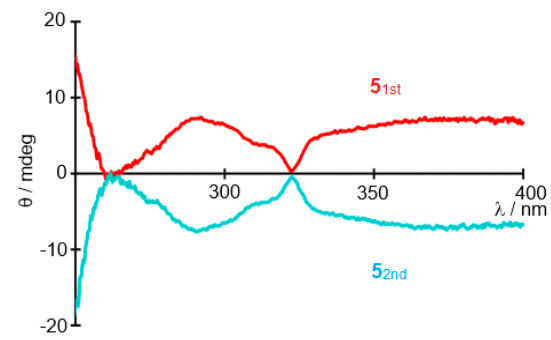

(a)

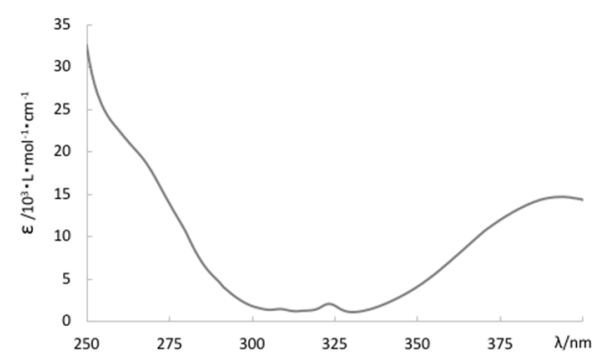

(b)

Figure 7. (a) Circular dichroism spectra of enantiomerically pure rotaxanes $\boldsymbol{5}_{1 \mathrm{st}}$ (red) and $\boldsymbol{5}_{2 \text { nd }}$ (blue) in $\mathrm{CHCl}_{3}$ at room temperature $\left(\left[5_{1 \mathrm{st}}\right]=90.7 \mu \mathrm{M},\left[5_{2 \text { nd }}\right]=94.6 \mu \mathrm{M}\right.$, cell length $\left.=1.0 \mathrm{~cm}\right)$; $(\mathbf{b}) \mathrm{Uv}$-visible spectrum of $\boldsymbol{5}_{2 \text { nd }}$ under same condition. 
In order to evaluate the enantioselective complexation ability of the rotaxane, titration experiments of rotaxane $\mathbf{5}_{2 \text { nd }}$ with $(R)$-phenylglycinol (PGO) and (S)-PGO were carried out. Because chiral host molecules with 2,4-dinitrophenylazophenol moiety produce ammonium phenolate salts in complexation with PGO accompanying large spectral change in UV-visible region with clear color change [22], there is a considerable chemical shift change of ${ }^{1} \mathrm{H}$ NNR signals assigned to the aromatic protons at low magnetic field.

As shown in Figure 8, spectral and color changes of $\mathrm{CHCl}_{3}$ solution of $\mathbf{5}_{2 \text { nd }}$ and $(R)$-PGO were observed. This observation shows that the extent of color change is clear enough as a sensor for naked eyes, and that the obtained rough extent of binding constant was around $100 \mathrm{~L} \mathrm{~mol}^{-1}$. Then, ${ }^{1} \mathrm{H} \mathrm{NMR}$ titration was revealed to be suitable for precise evaluation [52,53] of complexation ability of host 5 with PGO. The ${ }^{1} \mathrm{H}$ NMR titration was carried out with $\mathbf{5}_{2 \text { nd }}$ and enantiomeric pair of amines $(S)$ - and (R)-PGO in chloroform. Experimental details are filed in Supplementary Materials and Appendixs A-D. As shown in Table 1 , the determined binding constants of $5_{2 \text { nd }}$ were $(8.47 \pm 0.40) \times 10^{1} \mathrm{~L} \mathrm{~mol}^{-1}$ for $(S)$-PGO and $(1.25 \pm 0.03) \times 10^{2} \mathrm{~L} \mathrm{~mol}^{-1}$ for $(R)-\mathrm{PGO}$, respectively. The ratio of binding constants $K_{R} / K_{S}$ was 1.48. Generally speaking, as perspectives with the ratio of binding constant, it is not enough for application as chirality indicator $[19,53]$, but is promising for application as a chiral selector of a stationary phase for a chiral chromatography [54].

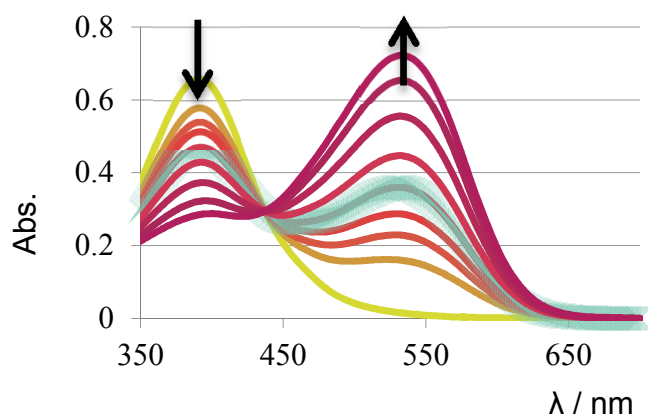

(a)

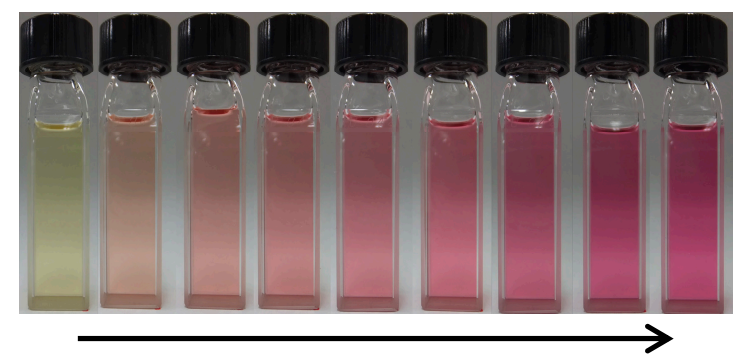

(b)

Figure 8. UV-Vis spectral and color changes of rotaxane $5_{2 \text { nd }}$ with (R)-PGO in $\mathrm{CHCl}_{3}$ : (a) UV-Vis spectra and (b) corresponding pictures: rotaxane $5(41.5 \mu \mathrm{M})((\mathbf{a})$ yellow line and (b) yellow solution) and same solutions containing different $(R)$-PGO concentration $899 \mu \mathrm{M}, 1.35,1.87,2.62,3.75,5.99,8.99$, and $12.7 \mathrm{mM}$ in order shown by arrows at $17^{\circ} \mathrm{C}$.

Table 1. Binding constants of $\mathbf{5}_{2 \text { nd }}$ for PGO obtained by ${ }^{1} \mathrm{H}$ NMR titration experiments.

\begin{tabular}{ccc}
\hline Guests & $\boldsymbol{K}_{\mathbf{L} ~ \mathbf{~ m o l}^{-\mathbf{1}}}$ & $\boldsymbol{K}_{\mathbf{R}} / \boldsymbol{K}_{\mathbf{S}}$ \\
\hline$(R)-\mathrm{PGO}$ & $(1.25 \pm 0.03) \times 10^{2}$ & \\
$(S)-\mathrm{PGO}$ & $(8.47 \pm 0.40) \times 10^{1}$ & 1.48 \\
\hline
\end{tabular}

In $\mathrm{CDCl}_{3}$ at $30^{\circ} \mathrm{C}, 400 \mathrm{MHz}$.

\section{Conclusions}

We developed an efficient method of rotaxane synthesis based on an aminolysis of a prerotaxane, which proceeded with excellent selectivities and chemical yields. We also found that the rotaxane with mechanical chirality has complexation ability against chiral amine PGO, with high enough enantioselectivity to be applied as a chiral selector of the chiral stationary phase for chiral chromatography. 
Supplementary Materials: The followings are available online at www.mdpi.com/2073-8994/10/1/20/s1, Table S1: Tabulated ${ }^{1} \mathrm{H}$ NMR titration data of Rotaxane $5_{2 \text { nd }}$ with $(R)$-PGO in $\mathrm{CDCl}_{3}$ at $30{ }^{\circ} \mathrm{C}$, Figure $\mathrm{S} 1$ : ${ }^{1} \mathrm{H}$ NMR titration curve for the complexation of Rotaxane $5_{2 \text { nd }}$ with $(R)-\mathrm{PGO}$ at $30{ }^{\circ} \mathrm{C}$, Table S2: Tabulated ${ }^{1} \mathrm{H}$ NMR titration data of Rotaxane $5_{2 \text { nd }}$ with (S)-PGO in $\mathrm{CDCl}_{3}$ at $30{ }^{\circ} \mathrm{C}$, Figure S2. ${ }^{1} \mathrm{H}$ NMR titration curve for the complexation of Rotaxane $5_{2 \text { nd }}$ with $(S)$-PGO at $30{ }^{\circ} \mathrm{C}$.

Acknowledgments: This work was partly supported by a Grant-in-Aid for Scientific Research from the Ministry of Education, Culture, Sports, Science, and Technology of Japan.

Author Contributions: Keiji Hirose conceived and designed the experiments; Masaya Ukimi, Shota Ueda, Chie Onoda, Ryohei Kano, Kyosuke Tsuda and Yuko Hinohara performed the experiments; Yoshito Tobe contributed for scientific guide; Masaya Ukimi, Shota Ueda, and Ryohei Kano analyzed the data; Keiji Hirose, Masaya Ukimi and Chie Onoda wrote the paper.

Conflicts of Interest: The authors declare no conflict of interest. The founding sponsors had no role in the design of the study; in the collection, analyses, or interpretation of data; in the writing of the manuscript, and in the decision to publish the results.

\section{Appendix A. General}

${ }^{1} \mathrm{H}$ NMR and ${ }^{13} \mathrm{C}$ NMR charts are filed here. Details of each chart are mentioned in Materials and Methods.

\section{Appendix B. ${ }^{1} \mathrm{H}$-and ${ }^{13} \mathrm{C}-\mathrm{NMR}$ Spectra of Ring 1}
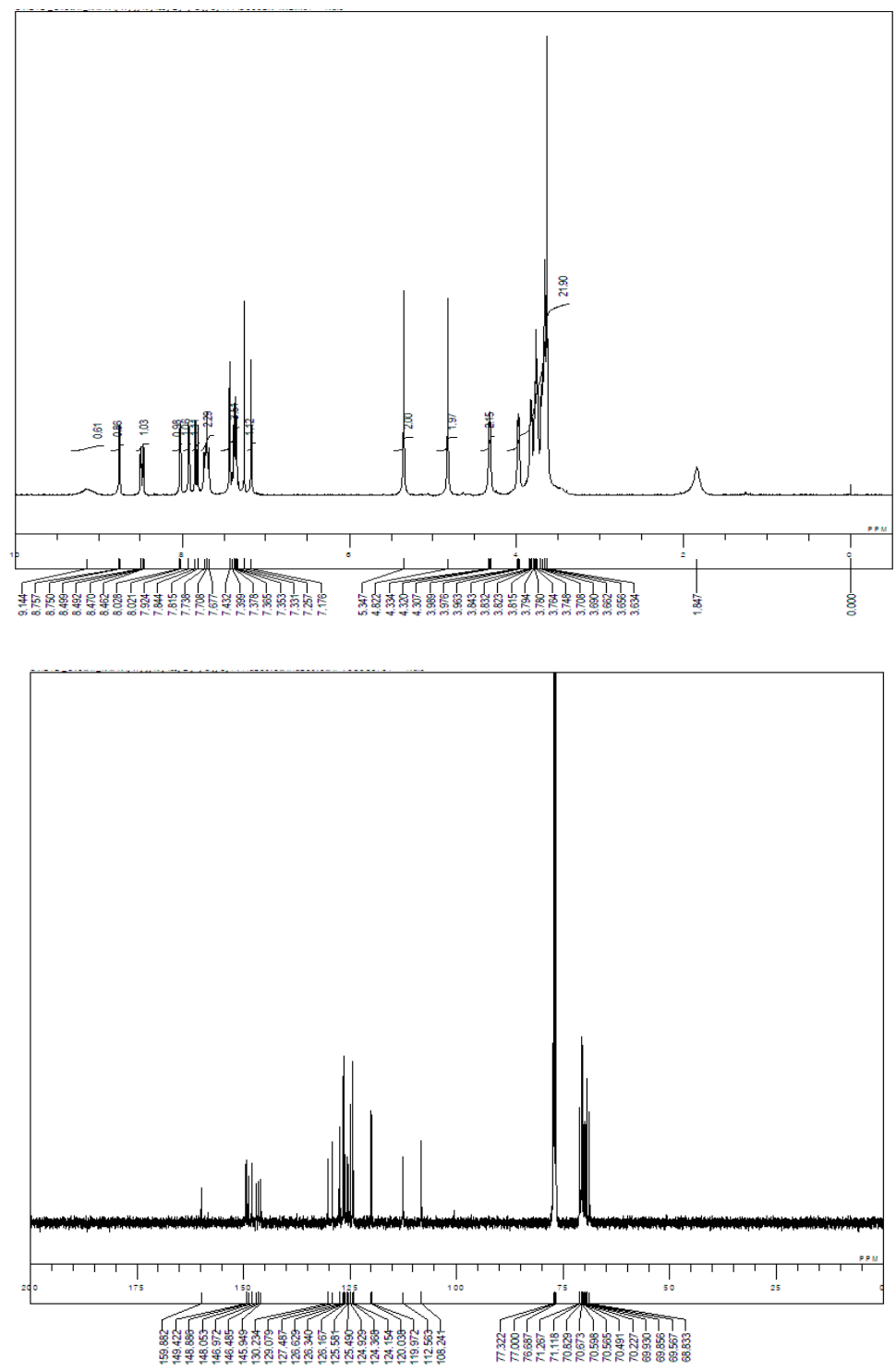
Appendix C. ${ }^{1} \mathrm{H}$-and ${ }^{13} \mathrm{C}-\mathrm{NMR}$ Spectra of Prerotaxanes 3
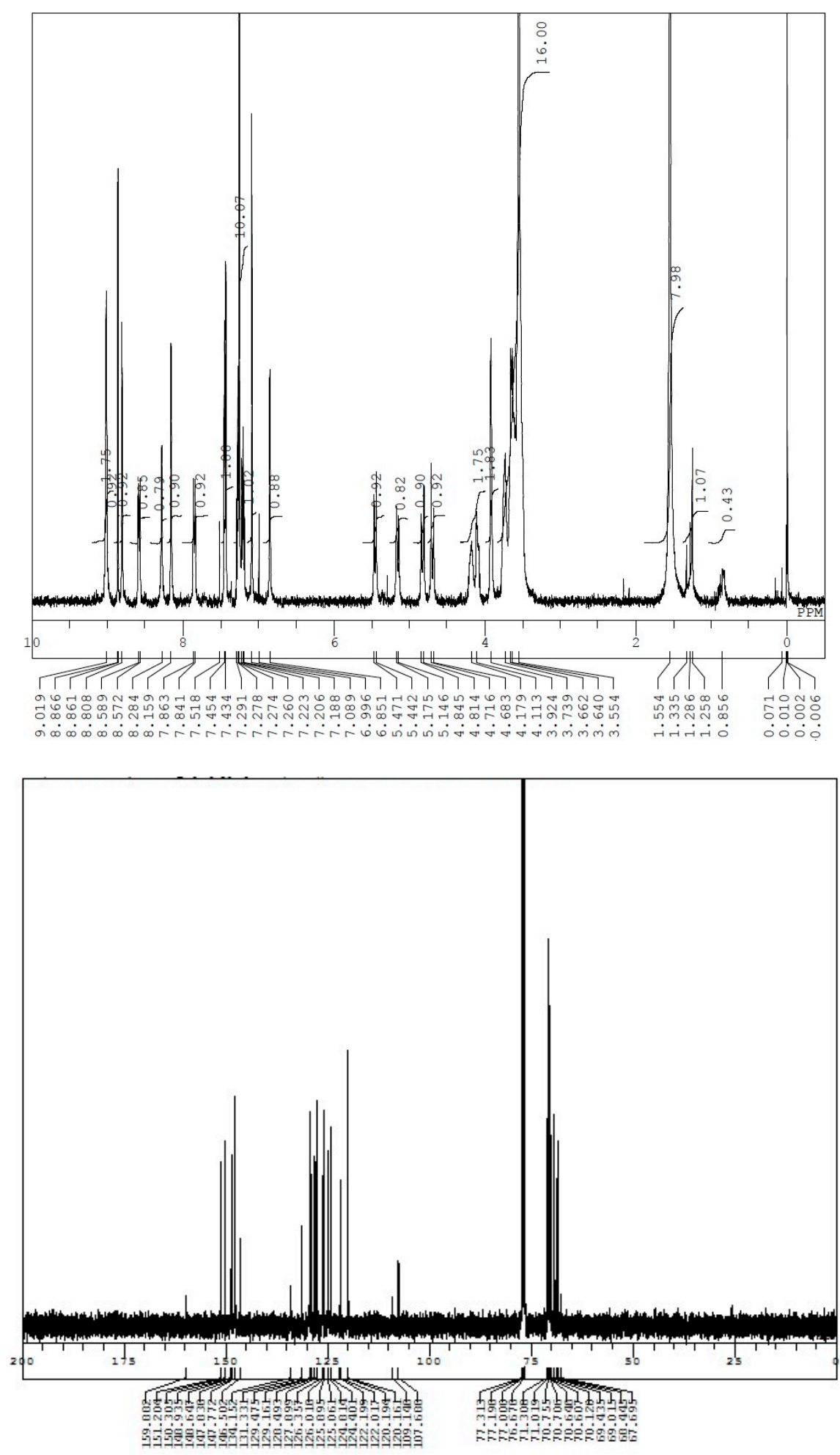
Appendix D. ${ }^{1} \mathrm{H}$-and ${ }^{13} \mathrm{C}-\mathrm{NMR}$ Spectra of Rotaxanes 5
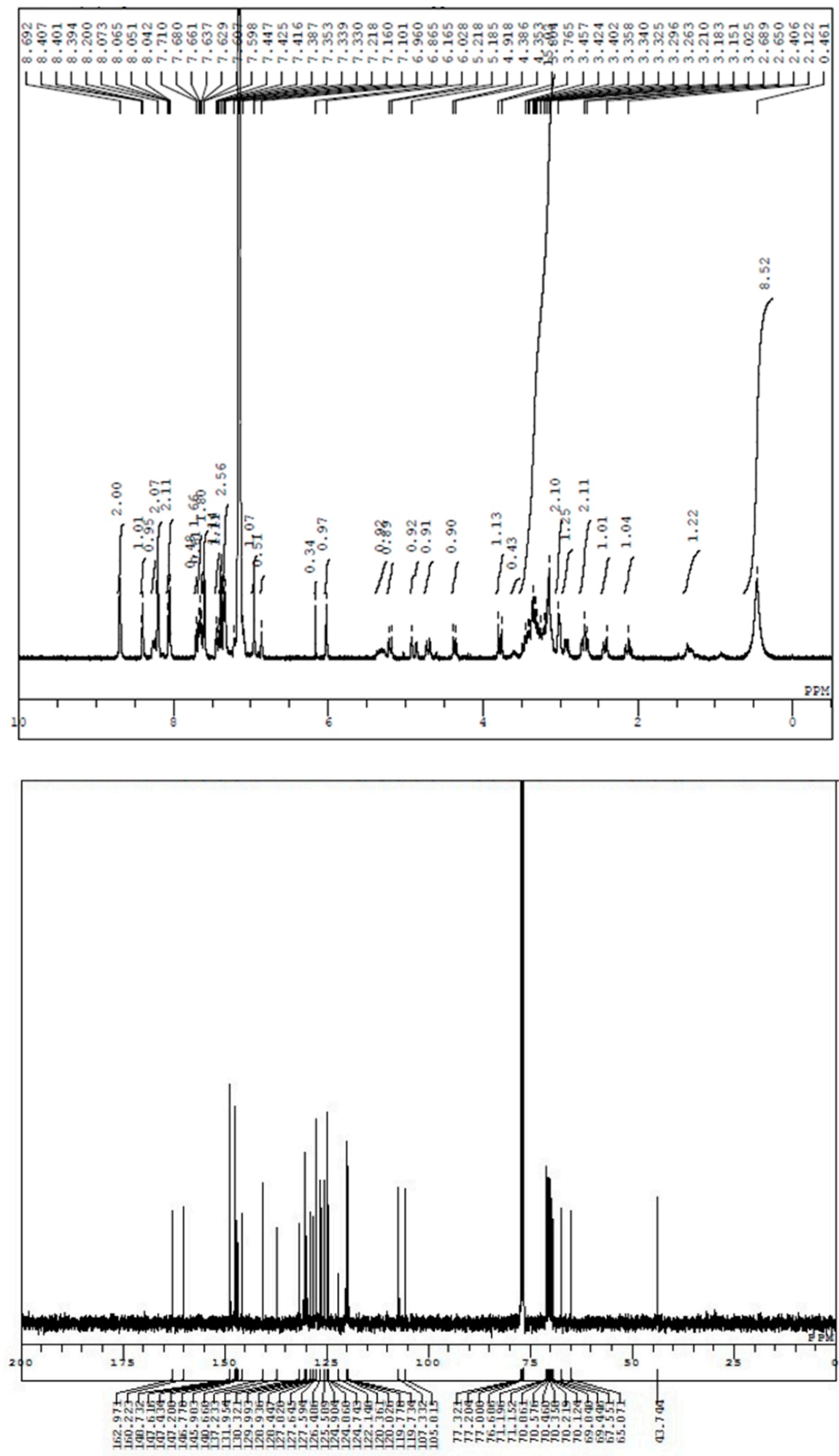

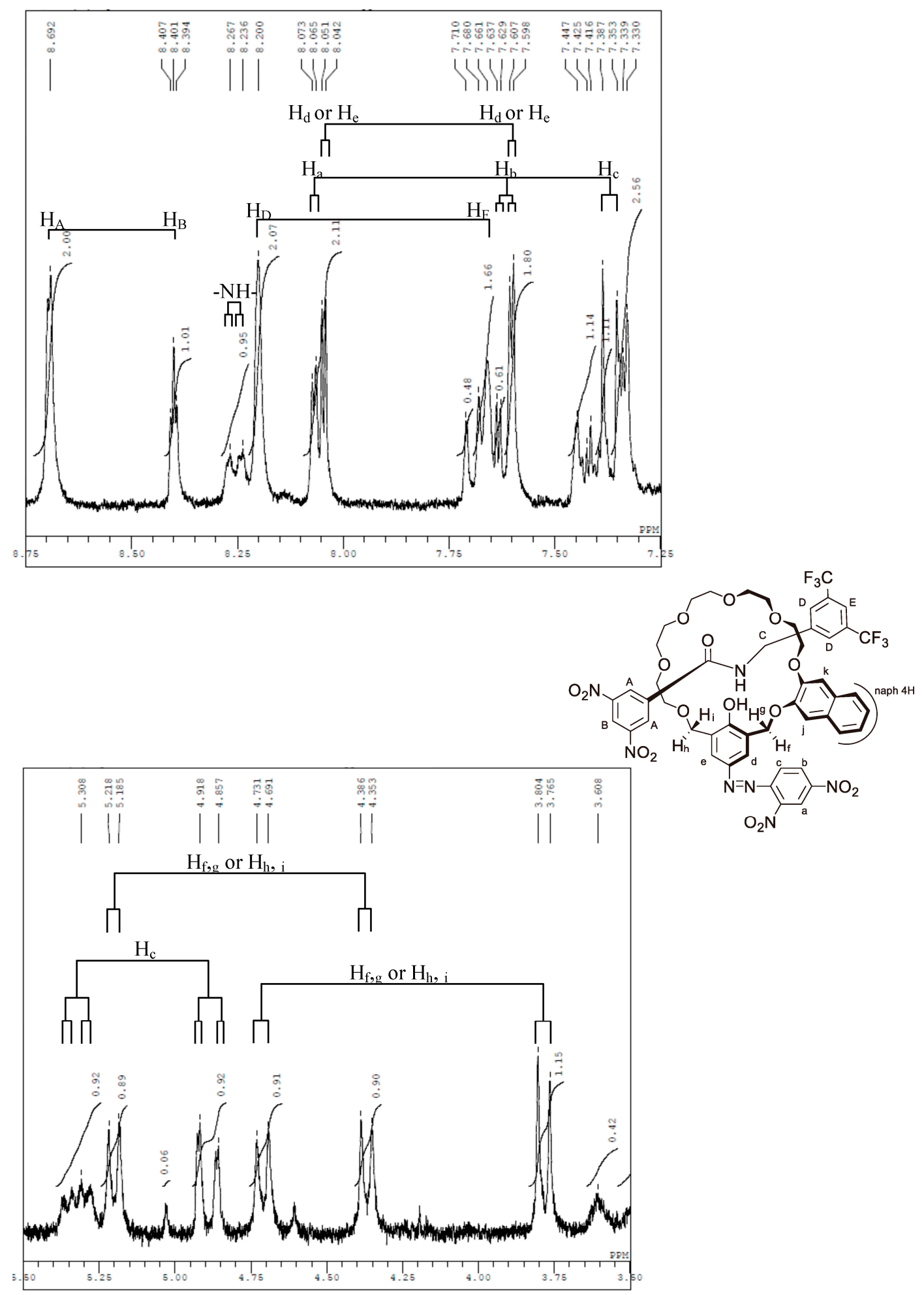

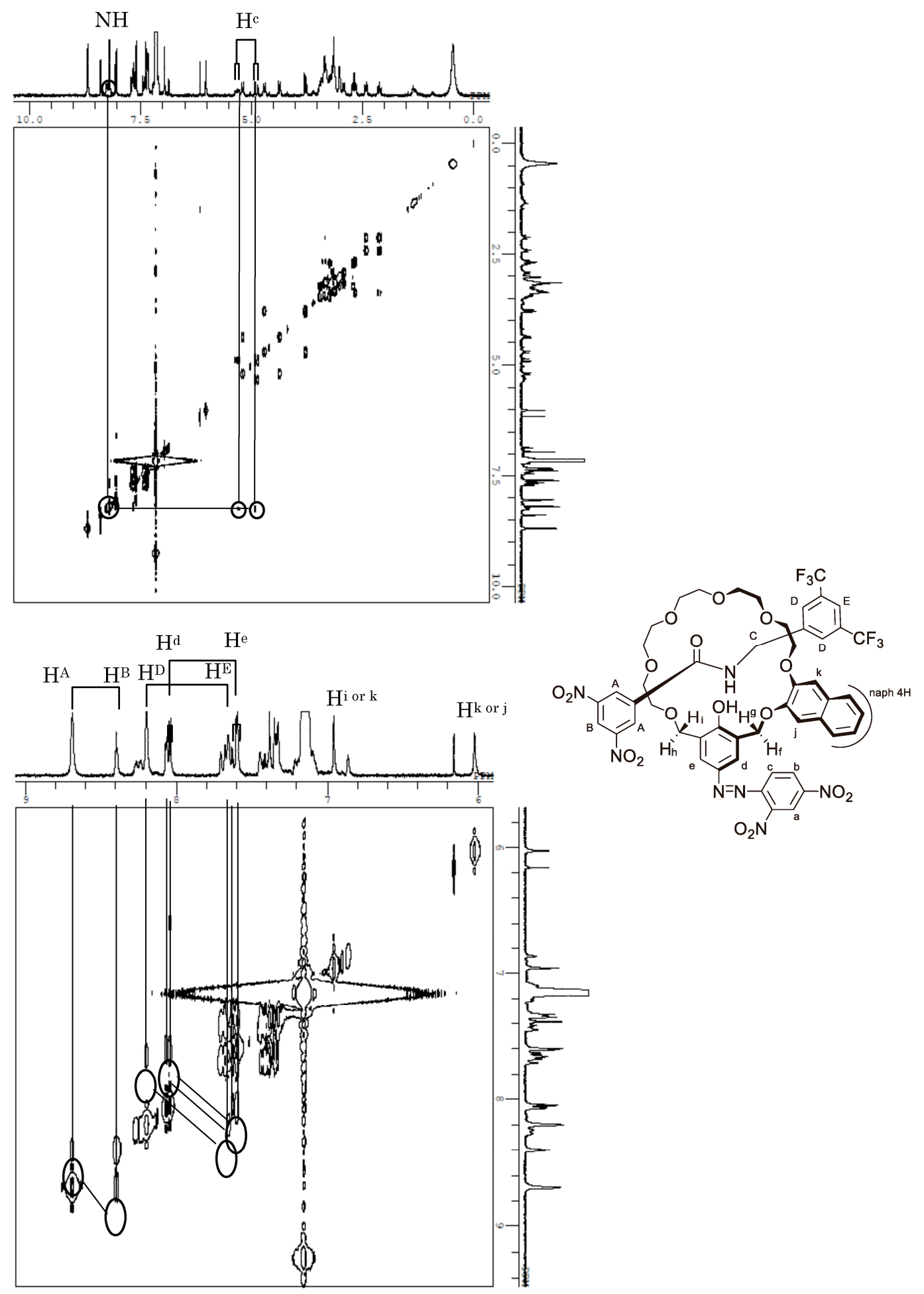

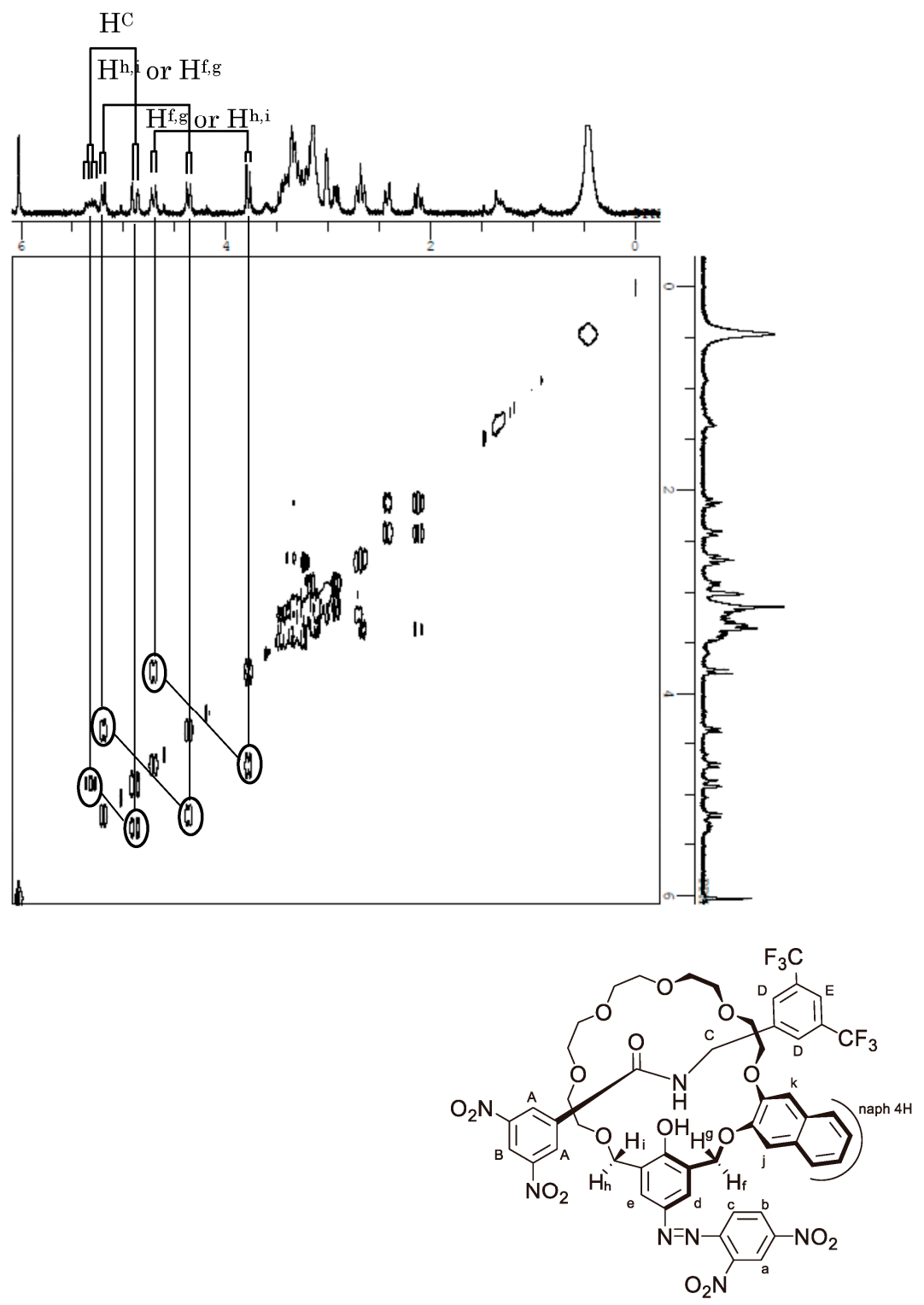

\section{References and Note}

1. Pedersen, C.J. The discovery of crown ethers. Science 1988, 241, 536-540. [CrossRef] [PubMed]

2. Cram, D.J. The design of molecular hosts, guests, and their complexes. J. Incl. Phenom. 1988, 6, $397-413$. [CrossRef]

3. Lehn, J.M. Supramolecular chemistry—Scope and perspectives molecules, supermolecules, and molecular devices. Angew. Chem. Int. Ed. 1988, 27, 89-112. [CrossRef]

4. Takagi, M.; Nakamura, H. Analytical application of functionalized crown ether-metal complexes. J. Coord. Chem. 1986, 15, 53-82. [CrossRef]

5. Nakashima, K.; Nagaoka, Y.; Nakatsuji, S.I.; Kaneda, T.; Tanigawa, I.; Hirose, K.; Misumi, S.; Akiyama, S. Fluorescence reactions of "crowned" benzothiazolylphenols with alkali and alkaline earth metal ions and their analytical applications. Bull. Chem. Soc. Jpn. 1987, 60, 3219-3223. [CrossRef]

6. Desilva, A.P.; Gunaratne, H.Q.N.; Gunnlaugsson, T.; Huxley, A.J.M.; Mccoy, C.P.; Rademacher, J.T.; Rice, T.E. Signaling recognition events with fluorescent sensors and switches. Chem. Rev. 1997, 97, 1515-1566. [CrossRef]

7. Hisamoto, H. Ion-selective optodes: Current developments and future prospects. Trac-Trends Anal. Chem. 1999, 18, 513-524. [CrossRef] 
8. Stradiotto, N.R.; Yamanaka, H.; Zanoni, M.V.B. Electrochemical sensors: A powerful tool in analytical chemistry. J. Braz. Chem. Soc. 2003, 14, 159-173. [CrossRef]

9. Faridbod, F.; Ganjali, M.R.; Dinarvand, R.; Norouzi, P.; Riahi, S. Schiff's bases and crown ethers as supramolecular sensing materials in the construction of potentiometric membrane sensors. Sensors 2008, 8 , 1645-1703. [CrossRef] [PubMed]

10. Sogah, G.D.Y.; Cram, D.J. Total chromatographic optical resolutions of alpha-amino acid and ester salts through chiral recognition by a host covalently bound to polystyrene resin. J. Am. Chem. Soc. 1976, 98, 3038-3041. [PubMed]

11. Machida, Y.; Nishi, H.; Nakamura, K.; Nakai, H.; Sato, T. Enantiomer separation of amino compounds by a novel chiral stationary phase derived from crown ether. J. Chromatogr. A 1998, 805, 85-92. [CrossRef]

12. Hyun, M.H.; Jin, J.S.; Lee, W.J. Liquid chromatographic resolution of racemic amino acids and their derivatives on a new chiral stationary phase based on crown ether. J. Chromatogr. A 1998, 822, 155-161. [CrossRef]

13. Maier, N.M.; Franco, P.; Lindner, W. Separation of enantiomers: Needs, challenges, perspectives. J. Chromatogr. A 2001, 906, 3-33. [CrossRef]

14. Hirose, K.; Nakamura, T.; Nishioka, R.; Ueshige, T.; Tobe, Y. Preparation and evaluation of novel chiral stationary phases covalently bound with chiral pseudo-18-crown-6 ethers. Tetrahedron Lett. 2003, 44, 1549-1551. [CrossRef]

15. Hirose, K.; Jin, Y.; Nakamura, T.; Nishioka, R.; Ueshige, T.; Tobe, Y. Chiral stationary phase covalently bound with a chiral pseudo-18-crown-6 ether for enantiomer separation of amino compounds using a normal mobile phase. Chirality 2005, 17, 142-148. [CrossRef] [PubMed]

16. Cram, D.J.; Cram, J.M. Design of complexes between synthetic hosts and organic guests. Acc. Chem. Res. 1978, 11, 8-14. [CrossRef]

17. Cram, D.J.; Trueblood, K.N. Concept, structure, and binding in complexation. Top. Curr. Chem. 1981, 98, 43-106.

18. Wenzel, T.J.; Freeman, B.E.; Sek, D.C.; Zopf, J.J.; Nakamura, T.; Jin, Y.; Hirose, K.; Tobe, Y. Chiral recognition in NMR spectroscopy using crown ethers and theirYtterbium(III) complexes. Anal. Bioanal. Chem. 2004, 378, 1536-1547. [CrossRef] [PubMed]

19. Kaneda, T.; Hirose, K.; Misumi, S. Chiral azophenolic acerands: Color indicator to judge the absolute configuration of chiral amines. J. Am. Chem. Soc. 1989, 111, 742-743. [CrossRef]

20. Hirose, K.; Goshima, Y.; Wakebe, T.; Tobe, Y.; Naemura, K. Supramolecular method for the determination of absolute configuration of chiral compounds: Theoretical derivatization and a demonstration for phenolic crown ether-2-amino-1-ethanol system. Anal. Chem. 2007, 79, 6295-6302. [CrossRef] [PubMed]

21. Kubo, Y.; Maeda, S.; Tokita, S.; Kubo, M. Colorimetric chiral recognition by a molecular sensor. Nature 1996, 382, 522-524. [CrossRef]

22. Naemura, K.; Takeuchi, S.; Hirose, K.; Tobe, Y.; Kaneda, T.; Sakata, Y. Preparation and enantiomer recognition behaviour of azophenolic crown ethers containing cis-cyclohexane-1,2-diol as the chiral centre. J. Chem. Soc. Perkin Trans. 1995, 213-219. [CrossRef]

23. Van Delden, R.A.; Feringa, B.L. Color indicators of molecular chirality based on doped liquid crystals. Angew. Chem. Int. Ed. 2001, 40, 3198-3200. [CrossRef]

24. Kim, H.N.; Guo, Z.; Zhu, W.; Yoon, J.; Tian, H. Recent progress on polymer-based fluorescent and colorimetric chemosensors. Chem. Soc. Rev. 2011, 40, 79-93. [CrossRef] [PubMed]

25. Sawada, M.; Takai, Y.; Yamada, H.; Hirayama, S.; Kaneda, T.; Tanaka, T.; Kamada, K.; Mizooku, T.; Takeuchi, S.; Ueno, K.; et al. Chiral recognition in host-guest complexation determined by the enantiomer-labeled guest method using fast atom bombardment mass spectrometry. J. Am. Chem. Soc. 1995, 117, 7726-7736. [CrossRef]

26. Sawada, M.; Takai, Y.; Yamada, H.; Nishida, J.; Kaneda, T.; Arakawa, R.; Okamoto, M.; Hirose, K.; Tanaka, T.; Naemura, K. Chiral amino acid recognition detected by electrospray ionization (ESI) and fast atom bombardment (FAB) mass spectrometry (MS) coupled with the enantiomer labelled (EL) guest method. J. Chem. Soc. Perkin Trans. 1998, 2, 701-710. [CrossRef]

27. Finn, M.G. Emerging methods for the rapid determination of enantiomeric excess. Chirality 2002, 14, 534-540. [CrossRef] [PubMed] 
28. Hembury, G.A.; Borovkov, V.V.; Inoue, Y. Chirality-sensing supramolecular systems. Chem. Rev. 2008, 108, 1-73. [CrossRef] [PubMed]

29. Jo, H.H.; Lin, C.-Y.; Anslyn, E.V. Rapid optical methods for enantiomeric excess analysis: From enantioselective indicator displacement assays to exciton-coupled circular dichroism. Acc. Chem. Res. 2014, 47, 2212-2221. [CrossRef] [PubMed]

30. Sauvage, J.P. Interlacing molecular threads on transition-metals: Catenands, catenates, and knots. Acc. Chem. Res. 1990, 23, 319-327. [CrossRef]

31. Blanco, M.J.; Jimenez, M.C.; Chambron, J.C.; Heitz, V.; Linke, M.; Sauvage, J.P. Rotaxanes as new architectures for photoinduced electron transfer and molecular motions. Chem. Soc. Rev. 1999, 28, 293-305. [CrossRef]

32. Kawasaki, H.; Kihara, N.; Takata, T. High yielding and practical synthesis of rotaxanes by acylative end-capping catalyzed by tributylphosphine. Chem. Lett. 1999, 10, 1015-1016. [CrossRef]

33. Schalley, C.A.; Weilandt, T.; Bruggemann, J.; Vögtle, F. Hydrogen-bond-mediated template synthesis of rotaxanes, catenanes, and knotanes. In Templates in Chemistry I; Springer: Berlin/Heidelberg, Germany, 2004; pp. 141-200. [CrossRef]

34. Arico, F.; Badjic, J.D.; Cantrill, S.J.; Flood, A.H.; Leung, K.C.F.; Liu, Y.; Stoddart, J.F. Templated synthesis of interlocked molecules. In Templates in Chemistry II; Springer: Berlin/Heidelberg, Germany, 2005; pp. $203-259$.

35. Narita, M.; Yoon, I.; Aoyagi, M.; Goto, M.; Shimizu, T.; Asakawa, M. Transition metal(II)-salen and -salophen macrocyclic complexes for rotaxane formation: Syntheses and crystal structures. Eur. J. Inorg. Chem. 2007, 4229-4237. [CrossRef]

36. Harrison, I.T.; Harrison, S. Synthesis of a stable complex of a macrocycle and a threaded chain. J. Am. Chem. Soc. 1967, 89, 5723-5724. [CrossRef]

37. Schill, G.; Zollenko, H. Rotaxane compounds 1. Liebigs Ann. Chem. 1969, 721, 53-74. [CrossRef]

38. Hiratani, K.; Suga, J.; Nagawa, Y.; Houjou, H.; Tokuhisa, H.; Numata, M.; Watanabe, K. A new synthetic method for rotaxanes via tandem claisen rearrangement, diesterification, and aminolysis. Tetrahedron Lett. 2002, 43, 5747-5750. [CrossRef]

39. Hirose, K.; Nishihara, K.; Harada, N.; Nakamura, Y.; Masuda, D.; Araki, M.; Tobe, Y. Highly selective and high-yielding rotaxane synthesis via aminolysis of prerotaxanes consisting of a ring component and a stopper unit. Org. Lett. 2007, 9, 2969-2972. [CrossRef] [PubMed]

40. Sauvage, J.P. From chemical topology to molecular machines (nobel lecture). Angew. Chem. Int. Ed. 2017, 56, 11080-11093. [CrossRef] [PubMed]

41. Stoddart, J.F. Mechanically interlocked molecules (MIMS)-molecular shuttles, switches, and machines (nobel lecture). Angew. Chem. Int. Ed. 2017, 56, 11094-11125. [CrossRef] [PubMed]

42. Feringa, B.L. The art of building small: From molecular switches to motors (nobel lecture). Angew. Chem. Int. Ed. 2017, 56, 11059-11078. [CrossRef] [PubMed]

43. Bordoli, R.J.; Goldup, S.M. An efficient approach to mechanically planar chiral rotaxanes. J. Am. Chem. Soc. 2014, 136, 4817-4820. [CrossRef] [PubMed]

44. Goldup, S.M. Mechanical chirality a chiral catalyst with a ring to it. Nat. Chem. 2016, 8, 404-406. [CrossRef] [PubMed]

45. Nakazono, K.; Takata, T. Chiral interlocked molecule: Synthesis and function. J. Synth. Organ. Chem. Jpn. 2017, 75, 491-502. [CrossRef]

46. Bruns, C.J.; Stoddart, J.F.; Sauvage, J.-P.; Fujita, M. The Nature of the Mechanical Bond: From Molecules to Machines; Wiley: Hoboken, NJ, USA, 2017; pp. 471-554. ISBN 9781119044000.

47. Borsche, W. Ueber die beziehungen zwischen chinonhydrazonen und p-oxyazoverbindungen. (vierte abhandlung): Ueber die condensation von nitroderivaten des phenylhydrazins mit chinonen und chinonoximen der benzolreihe. Liebigs Ann. Chem. 1907, 357, 171-191. [CrossRef]

48. Naemura, K.; Asada, M.; Hirose, K.; Tobe, Y. Preparation and enantiomer recognition of chiral azophenolic crown ethers having three chiral barriers on each of the homotopic faces. Tetrahedron Asymmetry 1995, 6, 1873-1876. [CrossRef]

49. Naemura, K.; Takeuchi, S.; Sawada, M.; Ueno, K.; Hirose, K.; Tobe, Y.; Kaneda, T.; Sakata, Y. Synthesis of azophenolic crown ethers of cs symmetry incorporating cis-1-phenylcyclohexane-1,2-diol residues as a steric barrier and diastereotopic face selectivity in complexation of amines by their diastereotopic faces. J. Chem. Soc. Perkin Trans. 1995, 1, 1429-1435. [CrossRef] 
50. Berova, N.; Di Bari, L.; Pescitelli, G. Application of electronic circular dichroism in configurational and conformational analysis of organic compounds. Chem. Soc. Rev. 2007, 36, 914-931. [CrossRef] [PubMed]

51. Stoncius, S.; Bagdziunas, G.; Malinauskiene, J.; Butkus, E. A study of planar chromophores in dichromophoric molecules by circular dichroism spectroscopy. Chirality 2008, 20, 337-343. [CrossRef] [PubMed]

52. Hirose, K. A practical guide for the determination of binding constants. J. Incl. Phenom. 2001, 39, $193-209$. [CrossRef]

53. Hirose, K. Quantitative analysis of binding properties. In Analytical Methods in Supramolecular Chemistry; Schalley, C.A., Ed.; John Wiley \& Sons: Weinheim, Germany, 2012; pp. 27-66, ISBN 9783527644131.

54. Requirements for an application to a chromatography: Resolution factor $(\alpha)$ for baseline separation (Resolution $(\mathrm{R})>1.25$, 99.4\% separation) through normal size column (Number of theoretical plates $(\mathrm{N})=5000$ ) is around 1.10 (Retention factor $\left(\mathrm{k}^{\prime} 2\right)=10$ ) where ratio of binding constant corresponds to resolution factor $(\alpha)$ of HPLC separation on chiral column.

(C) 2018 by the authors. Licensee MDPI, Basel, Switzerland. This article is an open access article distributed under the terms and conditions of the Creative Commons Attribution (CC BY) license (http://creativecommons.org/licenses/by/4.0/). 